\title{
QTL for body weight and condition factor in Atlantic salmon (Salmo salar): comparative analysis with rainbow trout (Oncorhynchus mykiss) and Arctic charr (Salvelinus alpinus)
}

\author{
DP Reid ${ }^{1}$, A Szanto ${ }^{1}$, B Glebe ${ }^{2}$, RG Danzmann ${ }^{1}$ and MM Ferguson ${ }^{1}$ \\ ${ }^{1}$ Department of Zoology, University of Guelph, Guelph, Ontario, Canada N1G 2W1; ${ }^{2}$ Department of Fisheries and Oceans, St. Andrews \\ Biological Station, St. Andrews, NB, Canada E0G 2X0
}

\begin{abstract}
Genotypes at 91 microsatellite loci in three full-sib families were used to search for QTL affecting body weight (BW) and condition factor in North American Atlantic salmon (Salmo salar). More than one informative marker was identified on 16-18 linkage groups in each family, allowing at least one chromosomal interval to be analyzed per linkage group. Two significant QTL for BW on linkage groups AS-8 and AS-11, and four significant QTL for condition factor on linkage groups AS-2, AS-5, AS-11, and AS-14 were identified. QTL for both BW and condition factor were located on linkage
\end{abstract}

groups AS-1, 6, 8, 11, and 14 when considering both significant and suggestive QTL effects. The largest QTL effects for BW (AS-8) and for condition factor (AS-14) accounted for 20.1 and $24.9 \%$ of the trait variation, respectively. Three of the QTL for BW occur on linkage groups where similar effects have been detected on the homologous regions in either rainbow trout (Oncorhynchus mykiss) or Arctic charr (Salvelinus alpinus).

Heredity (2005) 94, 166-172. doi:10.1038/sj.hdy.6800590

Published online 13 October 2004

Keywords: Atlantic salmon; microsatellite loci; QTL; body size; condition factor; comparative linkage analysis

\section{Introduction}

Species-specific linkage maps can be used to examine the genetic architecture of many fitness traits through quantitative trait analysis. Quantitative traits such as body size in salmonid fishes have a continuous phenotypic distribution and are controlled by chromosomal regions known as quantitative trait loci (QTL) (Mackay, 2001). The effects of allele segregation at molecular markers throughout the genome can be used to determine the number and positions of QTL affecting a trait, and the magnitude of the QTL effect (Lynch and Walsh, 1998). Linkage maps have been developed for several salmonid fishes, including rainbow trout (RT; Oncorhynchus mykiss) (Young et al, 1998; Sakamoto et al, 2000; Nichols et al, 2003), Arctic charr (AC; Salvelinus alpinus) (Woram et al, 2004), and Atlantic salmon (Salmo salar) (Gilbey et al, 2004; Moen et al, 2004). Despite the extensive karyotypic rearrangements within the tetraploid salmonids (Hartley, 1987; Phillips and Rab, 2001) since their evolution from a diploid ancestor 25-100 MYA (Allendorf and Thorgaard, 1984), microsatellite markers have been able to identify chromosomal regions that are homologous across species (Woram et al, 2003). Comparison of homologous regions among species and recombination rate between common markers has

Correspondence: MM Ferguson, Department of Zoology, University of Guelph, Guelph, Ontario, Canada N1G 2W1.

E-mail:mmfergus@uoguelph.ca

Received 31 October 2003; accepted 21 July 2004; published online 13 October 2004 identified several putative inter-specific fusions, inversions, and translocations (Woram et al, 2004). These homologous regions do not always span entire linkage groups in that many linkage groups show homologies to multiple linkage groups in the other salmonid species. The linkage groups of Atlantic salmon are expected to show a higher occurrence of multiple homologies relative to other species because it has a lower diploid chromosome number and chromosome arm number than most salmonids (Phillips and Rab, 2001).

QTL studies in RT and AC have revealed a great deal about the genetic architecture of body size (Robison et al, 2001) and its association with other traits such as age at maturation (Martyniuk et al, 2003). For example, $\mathrm{O}^{\prime} \mathrm{Mal}-$ ley et al (2003) detected three QTL for body weight (BW) in backcross RT females at 2 years of age plus four suggestive QTL for this trait. As well, Martyniuk et al (2003) showed that QTL for precocious maturation mapped to similar regions to those for body size in one strain of RT. This covariation in QTL location may partially explain the observed phenotypic correlations observed between body size and precocious maturation in salmonid fishes (Wilson et al, 2003). Finally, comparative mapping with AC and RT has shown that some body size QTL are conserved across species (Somorjai et al, 2003).

We searched for QTL affecting BW and condition factor in three full-sib families of North American Atlantic salmon by searching for associations among variation at 91 microsatellite loci located on 16 linkage groups (Reid, 2003) and the BW or condition factor of 1-year-old fish. A comparison of homologous regions in 
the Atlantic salmon, RT, and AC linkage maps was used to determine the degree of conservation of body size QTL among species.

\section{Materials and methods}

\section{Genotypic and phenotypic data}

Genotypic data for 91 microsatellite loci (Appendix 1; appendix available at: www.uoguelph.ca/ rdanzman/ appendices/) in three full-sib families (designated family 192, family 168, and family 151) of Atlantic salmon were used. The products from an additional 67 loci were uninformative (no amplification product or lack of polymorphism). The progeny came from parents spawned in 1998 as part of the Atlantic Salmon Broodstock Development Program, in Chamcook, New Brunswick. All families in the program are presumed to originate from the St. John River, New Brunswick. The male and female parents were designated according to the family identification number (ie, 192 dam and 192 sire). More than one informative marker was identified on 11-14 linkage groups in each family, allowing at least one interval in those linkage groups to be analyzed in the QTL analysis, while single-marker analyses were conducted for unlinked markers (ie in the three test families) with known linkage group affinities (unpublished results). Fork length (FL) in $\mathrm{cm}$ and $\mathrm{BW}$ in $\mathrm{g}$ were measured in 46 progenies from each of the three families. Fulton's condition factor $(K)$, a measure of a fish's girth, was calculated as $\left(100 \times \mathrm{BW} \cdot \mathrm{FL}^{-3}\right)$ (Rikardsen and Elliot, 2000). The regression of $\log \mathrm{FL}^{3}$ onto $\log \mathrm{BW}$ was approximately linear $\left(r^{2} \geqslant 0.83\right.$ in all three families) and the coefficient of slope $(b)$ was approximately $3(b=2.89$ 3.35). Due to the high correlation between FL and BW, and in order to allow for comparisons to QTL for BW and $\mathrm{K}$ in RT (O'Malley et al, 2003) and AC (Somorjai et al, $2003)$, only BW and K were used for QTL analysis. The mean, standard deviation, and range $(R)$ for $\mathrm{BW}$ and $K$ within each family were: family $192(\mathrm{BW}=126.5 \pm 40.3 \mathrm{~g}$, $R=42.6-241.6, K=1.158 \pm 0.066, R=1.016-1.384) ;$ family $168(\mathrm{BW}=131.7 \pm 35.1 \mathrm{~g}, R=77-230.2, K=1.256 \pm 0.064$ ， $R=1.113-1.399) ;$ family $151 \quad(\mathrm{BW}=121.2 \pm 27.1 \mathrm{~g}, R=$ 60.6-172, $K=1.213 \pm 0.055, R=1.09-1.332$ ).

Sampling methods precluded the sexing of the progeny. Instead, paternal allelic segregation at Ssa202DU was used to divide the progeny into two sex types. Ssa202DU is tightly linked to the sex-determining locus (approx. $4 \mathrm{cM}$ ) on the Atlantic salmon linkage map (Woram et al, 2003) and paternal allelic segregation at Ssa202DU was assumed to result in a reasonable estimation of progeny sex type. Using allelic segregation at a sex-linked marker cannot identify males versus females, but puts fish into two categories that correspond to different sexes. The two sex types did not differ in BW or condition factor except in family $192(P=0.021)$. Given the minor effects detected, the QTL analyses were performed on the entire family sample.

\section{QTL detection}

Phase-corrected genotypes using linkage group marker orders were established using LINKMFEX and GENOVECT (Danzmann, 2001) prior to QTL analysis. QTL effects were tested for the segregating alleles from the sires and dams separately for each interval or marker.
Shapiro-Wilkins and Kolmogorov-Smirnov tests for normality found no deviation from a standard normal distribution for BW or FL in any family (data not shown).

Initially, QTL were detected within each linkage group separately using interval mapping (IM) with MultiQTL v.1.1 (Korol, 2001). A reduced model that assumed equal trait variance between genotypic classes was compared to a second model (general model) that assumed unequal variance between genotypic classes. The general model was accepted only if the LOD values generated by each model were significantly different. A linkage group-wide permutation test (1000 replicates) determined the significance of the maximum LOD value over the various intervals analyzed for each linkage group. Multiple IM (MIM) (implemented within MultiQTL) was then used to reduce the background genetic noise and the influence of other QTLs from other chromosomes. Although the results of both analyses are reported in tabular form for completeness, the results from the MIM analysis are used in the textual reporting and discussion of the results.

The results of O'Malley et al (2003) showed that the permutation test from interval analysis (above) was much more conservative than a single-marker analysis on identical data sets. O'Malley et al (2003) found that a $P$-value of 0.01 from the interval analysis permutation test was equivalent to a $P$-value of 0.001 from a singlemarker analysis and would pass a Bonferroni correction for multiple tests. Thus, we considered a $P$-value $\leqslant 0.01$ from the interval analysis permutation test as significant and $P$-values between 0.01 and 0.05 as suggestive of a QTL effect.

A single-point analysis was performed for all unlinked markers using the individual marker option in MultiQTL. The number of unlinked markers ranged from 4 to 14 in any mapping parent and allele segregation at each marker was tested for significant association with phenotypic variation in BW and/or K. As stated above, the $P$-values obtained in a single-marker analysis are less conservative than those obtained in the interval analysis. As a result, a $P$-value of 0.001 was considered significant when analyzing single markers.

\section{Results}

Significant or suggestive BW QTL were detected on eight linkage groups (AS-1, AS-6, AS-8, AS-10, AS-11, AS-14, AS-17, AS-28) based on five of the six parents analyzed (Figure 1; Table 1). A significant QTL, accounting for $20.1 \%$ of the variation in BW, was identified on AS-8 (Ssa401UoS) using the progeny of dam 151. A QTL with relatively strong effect was found on AS-11; Ssa417UoS alleles segregating from two sires (sires 151 and 192) accounted for either 12.4 or $11.7 \%$ of the proportion of experimental variation (PEV) in BW. Sire 151 also showed effects on AS-1. Evidence for a suggestive QTL influencing BW on AS-10 was evident with dam 151 following MIM analysis. However, dam 192 showed a marginal QTL effect on AS-10 $(P=0.053$; $\mathrm{PEV}=0.123)$ using IM supporting the results obtained with dam 151 (marginal data not shown).

Significant QTL for condition factor were detected on AS-14 (dam 192; 24.9\% of the variation), AS-11 (dam 151; $16.9 \%$ ), AS-5 (dam 151; 17.6\%), and AS-2 (dam 168; $17.6 \%$ ) (Table 2). Suggestive QTL effects for condition factor were found on AS-1 (sire 192; $11.9 \%$ of the 
variation), AS-4 (sire 168; 14.0\%), AS-6 (sire 151; 14.2\%), AS-7 (dam 168; 15.4\% from interval analysis), and AS-8 (dam 192; 8.0\%). Although an interval QTL detection method was used in all these analyses, the most significant and suggestive QTL effects for both traits showed zero recombination with one of the two microsatellite markers flanking the interval. The only exceptions were on AS-4 and AS-6 for K (Table 2), where the peak LOD score was located within the interval. All the four markers shown in AS-11 for BW QTL in sire 192 (Table 1) mapped to a single location with zero recombination. a

\section{b AS-8f}

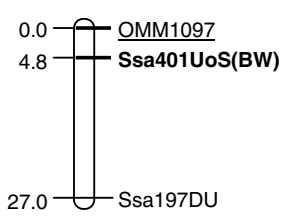

$\operatorname{Fam}(151+192)$

$0.0-\mathrm{B}$ - BHMS313A

Fam(151)

$0.0-\Theta$ Ssa197DU

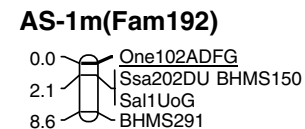

AS-1m(Fam168)

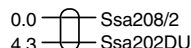

AS-1m(Fam151)

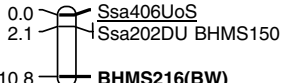

RT-31f

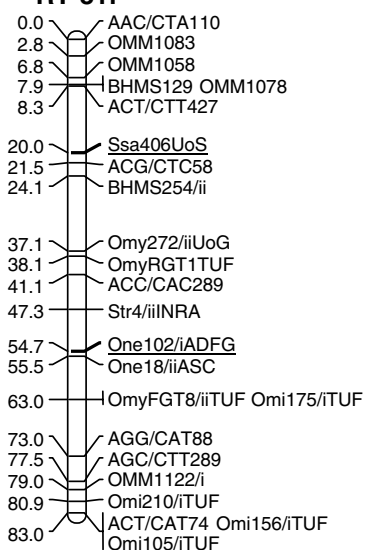

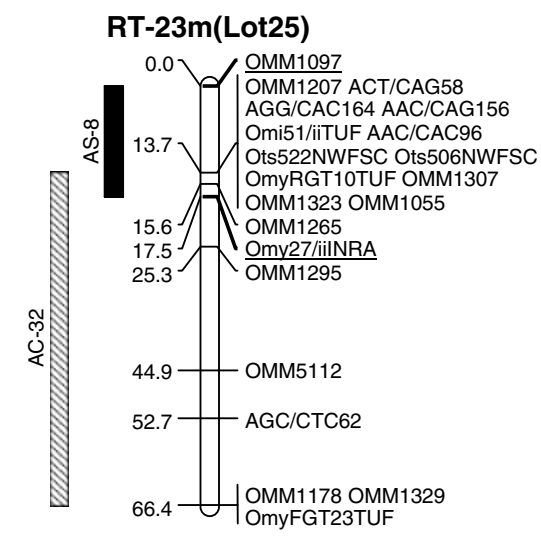

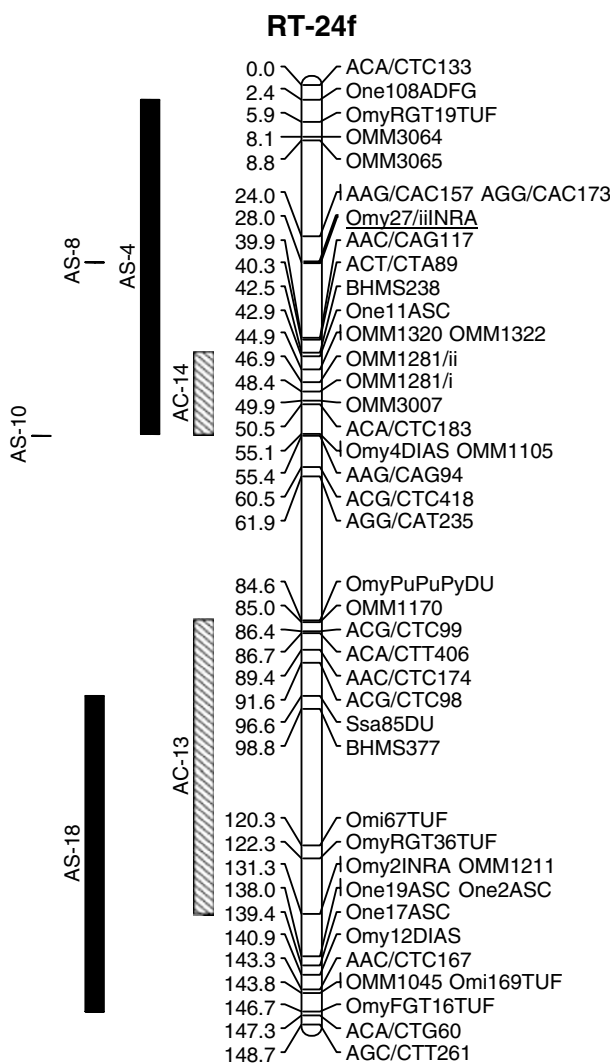

Figure 1 Comparative analysis of linkage groups that show QTL for BW in AS, RT, and AC. Fulton's condition factor (K) QTL are also indicated on the Atlantic salmon linkage groups that possess cross homologies to RT and AC linkage groups. QTL for BW and K detected in Atlantic salmon from this study are indicated in parentheses and in bold. Atlantic salmon maps are combined across mapping parents $(\mathrm{f}=$ female and $\mathrm{m}=$ male) unless indicated by a single-family designation. Please note: The assignment of marker BHMS313A to AS- 8 is based upon data presented in Moen et al (2004). Homologous affinities among linkage groups are depicted using the RT linkage map as a template (Danzmann et al, unpublished data) using a single mapping family (Lot25 or Lot44) or the combined map from both mapping panels. All homologous linkage groups are displayed on the same panels highlighting homologies to AS-1 (panel 1a), AS-8 (panel 1b), and AS-11 (panel 1c). Homologous markers that join the maps across species are underlined. QTL discussed in the text for RT are from Martyniuk et al (2003) and O'Malley et al (2003), and those for AC are from Somorjai (2001). The graphical figure was created using MAPCHART version 2.1 (Voorrips, 2002), using map information generated with LINKMFEX version 1.9 modules (Danzmann, 2001). 
C

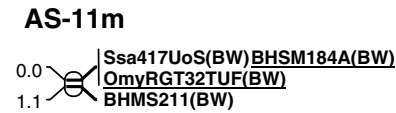

RT-9f

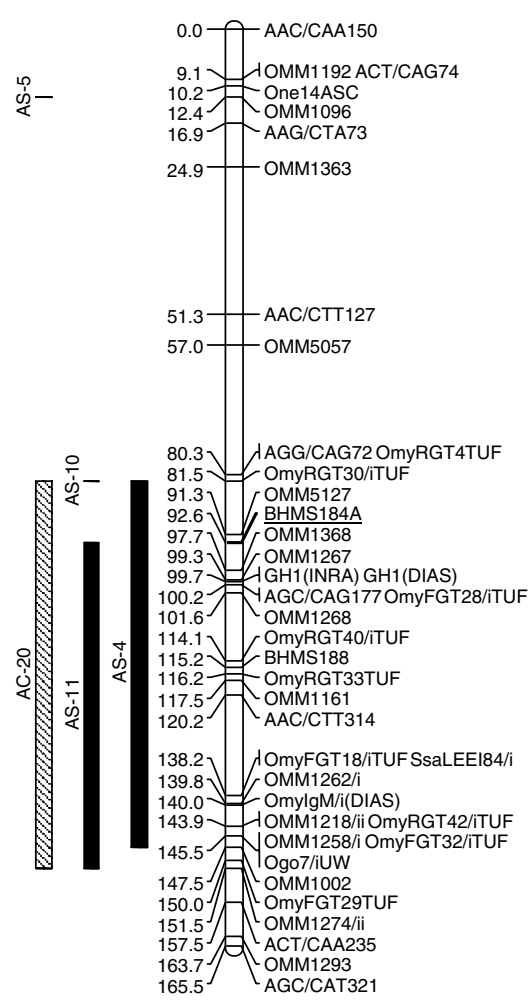

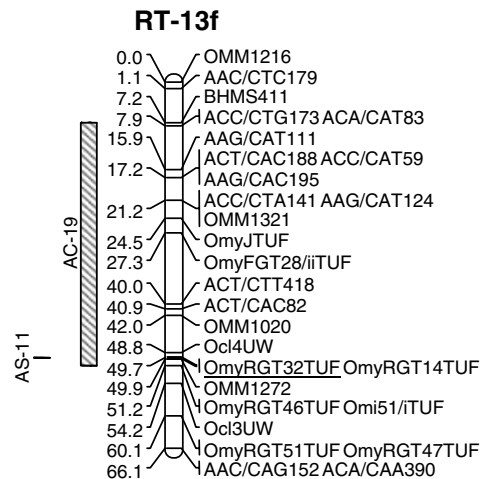

Figure 1 Continued.

Table 1 QTL analysis for body weight (BW) (g) in Atlantic salmon at 1 year of age using interval mapping and multiple interval mapping (see Materials and methods) as well as single-marker analysis

\begin{tabular}{|c|c|c|c|c|c|c|c|}
\hline \multirow[t]{2}{*}{$L G^{\mathrm{a}}$} & \multirow[t]{2}{*}{ Parent } & \multirow[t]{2}{*}{ Marker } & \multirow[t]{2}{*}{ Substitution effect $(g)^{\mathrm{b}}$} & \multicolumn{2}{|c|}{ Interval } & \multicolumn{2}{|c|}{ Multiple interval } \\
\hline & & & & $\mathrm{P}$ & $P E V^{c}$ & $\mathrm{P}$ & PEV \\
\hline 1 & 151 Sire & BHMS216 & 19.7 & 0.026 & 0.141 & 0.011 & 0.154 \\
\hline 6 & 192 Sire & BHMS382 & 26.7 & 0.042 & 0.126 & & \\
\hline 8 & 151 Dam & Ssa401Uos & 19.6 & 0.022 & 0.148 & 0.004 & 0.201 \\
\hline 10 & 151 Dam & SSOSL85 & 14.1 & & & 0.018 & 0.127 \\
\hline 11 & 192 Sire & $\begin{array}{l}\text { Ssa417UoS } \\
\text { OmyRGT32TUF } \\
\text { BHMS184A } \\
\text { BHMS211 }\end{array}$ & 31.5 & 0.008 & 0.170 & 0.013 & 0.117 \\
\hline 11 & 151 Sire & Ssa417UoS & 17.3 & 0.042 & 0.124 & 0.012 & 0.124 \\
\hline 14 & 168 Sire & BHMS111 & 24.5 & 0.043 & 0.140 & 0.045 & 0.122 \\
\hline 17 & 168 Dam & BHMS415 & 24.7 & 0.042 & 0.146 & 0.030 & 0.124 \\
\hline 28 & 192 Sire & Ssa405Uos & 29.2 & 0.023 & 0.137 & 0.033 & 0.091 \\
\hline
\end{tabular}

${ }^{a} \mathrm{LG}=$ linkage group.

${ }^{\mathrm{b}}$ Allelic substitution effect in grams.

${ }^{\mathrm{P}} \mathrm{PEV}=$ the proportion of experimental variation in $\mathrm{BW}$, accounted for by allele segregation at the specified marker or marker intervals

\section{Discussion}

We have located significant and suggestive QTL for the BW and condition factor $(K)$ of 1-year-old Atlantic salmon. Further, the mapping of QTL to four pairs of homeologous segments (AS-4/8, AS-4/10, AS-4/11, and AS-5/8) (Reid, 2003) provides some evidence that ancestrally duplicated chromosomes may retain similar gene function. Some of the QTL accounted for a large proportion ( $>20 \%$ ) of the phenotypic variation for either trait and suggests that a large proportion of the quantitative variation for $\mathrm{BW}$ and condition factor in Atlantic salmon is controlled by a few QTL with relatively large effect. This result is in keeping with previous studies with RT and AC (Martyniuk et al, 2003; O'Malley et al, 2003; Somorjai et al, 2003). However, the 
Table 2 QTL analysis for condition factor $(K)$ in Atlantic salmon at 1 year of age using interval mapping and multiple interval mapping as well as single-marker analysis (see Materials and methods)

\begin{tabular}{|c|c|c|c|c|c|c|c|}
\hline \multirow[t]{2}{*}{$L G^{\mathrm{a}}$} & \multirow[t]{2}{*}{ Parent } & \multirow[t]{2}{*}{ Marker } & \multirow[t]{2}{*}{ Substitution effect ${ }^{\mathrm{b}}$} & \multicolumn{2}{|c|}{ Interval } & \multicolumn{2}{|c|}{ Multiple interval } \\
\hline & & & & $\mathrm{P}$ & $P E V^{c}$ & $\mathrm{P}$ & PEV \\
\hline 1 & 192 Sire & One102ADFG & 0.046 & 0.034 & 0.135 & 0.034 & 0.119 \\
\hline 2 & 168 Dam & BHMS159 & 0.054 & 0.019 & 0.185 & 0.010 & 0.176 \\
\hline 4 & 168 Sire & $\begin{array}{l}\text { OMM1002 } \\
\text { BHMS177/i }\end{array}$ & 0.048 & 0.038 & 0.154 & 0.038 & 0.140 \\
\hline 5 & 151 Dam & Str58CNRS & 0.052 & $<0.001$ & 0.238 & 0.002 & 0.176 \\
\hline 6 & 151 Sire & $\begin{array}{l}\text { OtsG68UCD } \\
\text { BHMS382 }\end{array}$ & 0.042 & 0.011 & 0.164 & 0.013 & 0.142 \\
\hline 7 & 168 Dam & BHMS337 & 0.041 & 0.033 & 0.154 & & \\
\hline 8 & 192 Dam & BHMS313A & 0.038 & 0.045 & 0.102 & 0.016 & 0.080 \\
\hline 11 & 151 Dam & BHMS211 & 0.049 & $<0.001$ & 0.241 & 0.004 & 0.169 \\
\hline 14 & 192 Dam & OtsG249UCD & 0.066 & 0.005 & 0.267 & $<0.001$ & 0.249 \\
\hline
\end{tabular}

${ }^{a} \mathrm{LG}=$ linkage group.

bAllelic substitution effect for $K$.

${ }^{\mathrm{P}} \mathrm{PEV}=$ The proportion of experimental variation in $K$, accounted for by allele segregation at the specified microsatellite locus

statistical analyses used are biased towards detecting QTL with larger effect (Mackay, 2001). This results in an underestimation of the total number of genes affecting a trait. Further study including increasing marker density in the North American Atlantic salmon linkage map and additional QTL analysis with more families will allow for a more detailed study to determine the true number and magnitudes of effect of QTL affecting BW and K.

The detection of BW and K QTL on the same linkage groups (ie AS-1, AS-6, AS-8, AS-11, and AS-14) can be explained by either the linkage of two QTL (one for each trait), or the presence of a single QTL on each linkage group with pleiotropic effects. Previous studies have found that the genetic correlation between BW and condition factor is low at least in RT (Fishback et al, 2002). This suggests that the two traits have some degree of independence in genetic control and that different sets of genes might be involved. However, distinguishing between a single gene versus a gene cluster with individual QTL is difficult, given the linkage map density used in this study. The hypothesis of a single QTL with pleiotropic effects could be supported if the effects were localized to an interval with a high density of markers in a female parent. Unfortunately, one or both of the QTL effects were detected in a male parent in all the linkage groups where both BW and K QTL effects were observed, with the exception of AS-8. Since males show greatly reduced recombination rates compared to females (Johnson et al, 1987; Sakamoto et al, 2000), it is difficult to localize QTL positions based upon male genetic maps. On AS-8, however, the QTL for BW was localized to marker Ssa401UoS, while the QTL for K was localized to marker BHMS313A, which are unlinked on the female map (but are linked in the male map). This lack of linkage coupled with the detection of effects in female parents suggests that separate QTL for BW and K might exist on linkage group AS-8.

Three of the significant QTL for BW identified in Atlantic salmon that occur on linkage groups AS-1, AS-8, and AS-11 may be homologous to BW QTL regions in RT and AC (Somorjai, 2001; Martyniuk et al, 2003; O'Malley et al, 2003). AS-1 shows homology to a pair of RT chromosomes (RT-27 and RT-31) that represent ancestral homeologues (Woram et al, 2003). There is strong support for the existence of a BW QTL on linkage group RT-31 (Figure 1a). AS-1 shares three markers with RT-27 (OmyFGT8TUF, One18ASC, and One102ADFG) and four markers (OmyFGT8TUF, Str4INRA, One18ASC, and One102ADFG) with RT-31 (Woram et al, 2003). The QTL for BW identified in RT (OmyRGT1TUF) (Martyniuk et al, 2003) is only localized within the male map, making it difficult to ascertain the exact position of the QTL. In male mapping parents, this marker maps with either zero recombination or within $3 \mathrm{cM}$ with the markers on RT-31 (Sakamoto et al, 2000). Since the BW QTL in Atlantic salmon on AS-1 was also localized within the male map, we cannot directly compare homologies between the two species.

The BW QTL with the greatest effect in Atlantic salmon was detected in a region of AS-8 that shows homology to RT and AC linkage groups RT-24 and AC13, respectively (Somorjai, 2001; O'Malley et al, 2003). Unfortunately, the QTL regions in these studies were localized in the male mapping parent, making it difficult to ascertain the exact chromosomal location of the QTL region, for reasons discussed previously. A single marker, Ssa85DU, analyzed by Somorjai (2001) (present in the central region of RT-24 and on AC-13) (see Figure $1 \mathrm{~b}$ for inferred homologies), demonstrated a significant BW QTL effect in an AC male mapping parent. Due to the limited density of markers on the AC linkage map (Woram et al, 2004), homologous associations between Atlantic salmon and AC must be inferred from the similarities that each species has with RT. In comparisons with the RT map, it appears that the region of RT-24 containing Ssa85DU is homologous to AS-18 and not AS-8. However, the localization of the QTL position in AC remains imprecise since the effect was detected in a male mapping parent. Nonetheless, the data suggest that more than one BW QTL region resides within RT-24. Interestingly, homeologous affinities between $\mathrm{AC}-13$ and $\mathrm{AC}-14$ have been detected in $\mathrm{AC}$ (Woram et al, 2004), suggesting that RT-24 may also contain homeologous genome segments.

Of particular note is the apparent conservation of the strongest BW QTL in both Atlantic salmon and RT in similar chromosomal segments. The region of linkage group AS-8, where we detected the QTL (explaining 20\% 
of the variation in BW), appears to be syntenic with RT23/24 (RT-23/24 are putative homeologous chromosome pairs in RT) (Sakamoto et al, 2000; Nichols et al, 2003). AS8 shares homology to both RT-21 and 24. However, the region of AS-8 showing the BW QTL effect (ie marker Ssa401UoS) maps adjacent to marker OMM1097, and this latter marker localizes the QTL region to a homologous segment of RT-23/24. Additional research with our RT mapping panel has localized the strongest BW QTL in this species (explaining $34 \%$ of the variance in the trait) to the central region of male linkage group RT-24 (O'Malley et al, 2003).

The chromosomal segment on AS-11, where a BW QTL was detected (marked by OmyRGT32TUF), appears to be homologous to a segment of RT-13 and AC-19 where BW QTL have also been detected (Somorjai, 2001; O'Malley et al, 2003) (Figure 1c). Variation in BW was associated with allele segregation at OmyRGT14TUF, OmyRGT47TUF, and OmyRGT51TUF on RT-13 in RT (Martyniuk et al, 2003). Unfortunately, the low density of markers on AC19 does not allow us to relate these regions directly, but the presence of a marginal QTL effect on BW with OmyRGT46TUF on AC-19 in a male AC parent (Somorjai, 2001) does support the contention that at least one of these regions is conserved in $\mathrm{AC}$.

We cannot exclude the possibility that the BW QTL region detected on AS-11 is homologous to RT and AC linkage groups RT-9 and AC-20, respectively. Regions of RT-13 and RT-9 share homeology with one another in RT (Sakamoto et al, 2000) and this homeologous synteny is supported by the fact that markers showing homology to both RT-9 (marker BHMS184A) and RT-13 (marker OmyRGT32TUF) occur on AS-11. In addition, marker BHMS211, which maps to AS-11, is located on RT linkage group RT-2, and RT-2 and RT-9 share homeologous affinities. Marker BHMS184A maps proximally to growth hormone (Figure 1c) and BW QTL effects have been detected with markers mapping proximally to growth hormone on RT-9 (Martyniuk et al, 2003), suggesting a possible influence of this gene on the observed phenotypic distributions.

This study has identified chromosomal regions that appear to show conserved QTL effects on BW in three genera of salmonid fishes (Salmo, Oncorhynchus, and Salvelinus). Three of eight linkage groups examined showed evidence for conserved effects between Atlantic salmon and RT, while two regions may be conserved between Atlantic salmon and AC. Markers on AS-6, AS10, AS-14, AS-17, and AS-28 did not appear to share homologous BW QTL with RT or AC. It is important to note, however, that homologies among linkage groups among these species are largely unknown and, therefore, future research will likely identify additional interspecific homologies for growth QTL corresponding to these Atlantic salmon linkage groups. The detection of a greater number of conserved QTL effects in comparisons between Atlantic salmon and RT than with AC can probably be attributed to the greater marker density in the former two species.

\section{Acknowledgements}

We thank the Atlantic Salmon Broodstock Development Program for providing the fish. This work was supported by a Genomics Project from NSERC (Canada) and funds from the Huntsman Marine Sciences Centre (St. Andrews, New Brunswick).

\section{References}

Allendorf F, Thorgaard G (1984). Tetraploidy and the evolution of salmonid fishes. In: Turner B (ed) Evolutionary Genetics of Fishes. Plenum Press: New York pp 1-54.

Danzmann RG (2001). LINKMFEX: Linkage analysis package for outcrossed mapping families with male or female exchange of the mapping parent www.uoguelph.ca/ $\sim$ rdanzman/software.

Fishback AG, Danzmann RG, Ferguson MM, Gibson JP (2002). Estimates of genetic parameters and genotype by environment interactions for growth traits of rainbow trout (Oncorhychus mykiss) as inferred using molecular pedigree. Aquaculture 206: 137-150.

Gilbey J, Verspoor E, McLay A, Houlihan D (2004). A microsatellite linkage map for Atlantic salmon (Salmo salar). Animal Genet 35: 98-105.

Hartley S (1987). The chromosomes of salmonid fishes. Biol Rev Cambridge Philos Soc 62: 197-214.

Johnson KR, Wright JE, May B (1987). Linkage relationships reflecting ancestral tetraploidy in salmonid fish. Genetics 116 $579-591$.

Korol AB (2001). MultiQTL software v1.5. MultiQTL Ltd. Haifa, Israel www.multiqtl.com.

Lynch M, Walsh B (1998). Genetics and Analysis of Quantitative Traits. Sinauer Assoc. Inc.: Sunderland, MA, USA.

Mackay TFC (2001). The genetic architecture of quantitative traits. Ann Rev Genet 35: 303-339.

Martyniuk CJ, Perry GML, Moghadam HK, Ferguson MM, Danzmann RG (2003). The genetic architecture of correlations among growth-related traits and male age at maturation in rainbow trout (Oncorhynchus mykiss). J Fish Biol 63: 746-764.

Moen T, Hoyheim B, Munck H, Gomez-Raya L (2004). A linkage map of Atlantic salmon (Salmo salar) reveals an uncommonly large difference in recombination rate between the sexes. Animal Genet 35: 81-92.

Nichols KM, Young WP, Danzmann RG, Robison BD, Rexroad C, Noakes M et al (2003). A consolidated linkage map for rainbow trout (Oncorhychus mykiss). Animal Genet 34: 102-115.

O'Malley KG, Sakamoto T, Danzmann RG, Ferguson MM (2003). Quantitative trait loci for spawning date and body weight in rainbow trout: testing for conserved effects across ancestrally duplicated chromosomes. J Hered 94: 273-284.

Phillips RB, Rab P (2001). Chromosome evolution in the Salmonidae (Pisces): an update. Biol Rev 76: 1-25.

Reid DP (2003) Comparative linkage analysis of North American and European Atlantic salmon (Salmo salar) and detection of quantitative trait loci for body weight and condition factor. MSc Thesis, University of Guelph, ON, Canada.

Rikardsen AH, Elliot JM (2000). Variations in juvenile growth, energy allocation and life-history strategies of two populations of Arctic charr in north Norway. J Fish Biol 56: 328-346.

Robison BD, Wheeler PA, Sundin K, Thorgaard GH (2001). Composite interval mapping reveals a major locus influencing embryonic development rate in rainbow trout (Oncorhynchus mykiss). J Hered 92: 16-22.

Sakamoto T, Danzmann RG, Gharbi K, Howard P, Ozaki A, Kean Khoo $S$ et al (2000). A microsatellite linkage map of rainbow trout characterized by large sex-specific differences in recombination rates. Genetics 155: 1331-1345.

Somorjai IML (2001) Quantitative trait loci for fitness traits in Arctic charr: conservation in rainbow trout and correlations among traits. MSc Thesis, University of Guelph, ON, Canada.

Somorjai IML, Danzmann RG, Ferguson MM (2003). Distribution of temperature tolerance quantitative trait loci in Arctic 
charr (Salvelinus alpinus) and inferred homologies in rainbow trout (Oncorhynchus mykiss). Genetics 165: 1443-1456.

Voorrips RE (2002). MapChart. Software for the graphical presentation of linkage maps and QTL. J Hered 93: 77-78 Available at: www.joinmap.nl.

Wilson AJ, Hutchings JA, Ferguson MM (2003). Selective and genetic constraints on the evolution of body size in a stream dwelling salmonid fish. J Evol Biol 16: 584-594.

Woram RA, Gharbi K, Sakamoto T, Hoyheim B, Holm L-E, Naish K et al (2003). Comparative genome analysis of the primary sex-determining locus in salmonid fishes. Genome Res 13: 272-280.

Woram RA, McGowan C, Stout JA, Gharbi K, Ferguson MM, Hoyheim B et al (2004). A genetic linkage map for Arctic charr (Salvelinus alpinus): evidence for higher recombination rates and segregation distortion in hybrid versus pure strain mapping parents. Genome 47: 304-315.

Young W, Wheeler P, Coryell V, Keim P, Thorgaard G (1998). A detailed linkage map of rainbow trout using doubled haploids. Genetics 148: 839-850. 